\title{
CD36: a class B scavenger receptor involved in angiogenesis, atherosclerosis, inflammation, and lipid metabolism
}

\author{
Maria Febbraio, ${ }^{1,2}$ David P. Hajjar, ${ }^{2,3}$ and Roy L. Silverstein ${ }^{1,2}$ \\ ${ }^{1}$ Department of Medicine, Division of Hematology-Medical Oncology, \\ ${ }^{2}$ Center of Vascular Biology, and \\ ${ }^{3}$ Department of Pathology and Department of Biochemistry, Weill Medical College of Cornell University, \\ 1300 York Avenue, New York, New York, USA \\ Address correspondence to: Maria Febbraio, Department of Medicine, Division of Hematology-Medical Oncology, \\ Weill Medical College of Cornell University, 1300 York Avenue, Box 113, New York, New York 10021, USA. \\ Phone: (212) 746-2068; Fax: (212) 746-8866; E-mail: mjfebbra@med.cornell.edu.
}

J. Clin. Invest. 108:785-791 (2001). DOI:10.1172/JCI200114006.

CD36, identified more than a quarter of a century ago as a platelet integral membrane glycoprotein (glycoprotein IV), was until recently best known as a receptor for thrombospondin-1 (TSP-1). TSP-1 is found in ECMs and platelet $\alpha$ granules, and it participates in cell attachment, motility, and proliferation, as well as in modulation of protease activity, TGF- $\beta$ activation, neurite outgrowth, and angiogenesis (1). Initially, this receptor-ligand pair was shown to mediate interactions between platelets and monocytes, tumor cells, and matrix. Since then, CD36 has been implicated in multiple biological processes that define it as a multiligand scavenger receptor (see ref. 2 for review). These ligands appear remarkably diverse: In addition to TSP-1, they include long-chain fatty acids, modified LDL, retinal photoreceptor outer segments, Plasmodium falciparum malaria-parasitized erythrocytes, sickle erythrocytes, anionic phospholipids, apoptotic cells, and collagens I and IV. The biology of CD36 can be broadly divided in terms of functions that it mediates with or without TSP-1, but it is probable that it acts in concert with other proteins, such as fatty acid-binding proteins, caveola-associated proteins, integrins, cytoskeletal proteins, and signaling molecules, to effect its diverse functions.

\section{Molecular properties}

CD36 belongs to the class B scavenger receptor fami$l y$, which includes the receptor for selective cholesteryl ester uptake, scavenger receptor class B type I (SR-BI), and lysosomal integral membrane protein II (LIMP-II). The nucleotide sequence of the human cDNA predicts a protein of 471 amino acids and a molecular weight of $53 \mathrm{kDa}$ (3). The protein is heavily N-linked glycosylated, a modification that may provide proteins in this family some protection from degradation in proteinase-rich environments such as the lysosome and areas of inflammation or tissue damage. Within the carboxy-terminal segment of
CD36 is a region of 27 hydrophobic amino acids corresponding to a transmembrane domain. The aminoterminus has an uncleaved signal peptide, which is probably a second membrane-spanning domain. The predicted structure orients most of the protein extracellularly, except for two short (9-13 amino acids) cytoplasmic tails which can be palmitoylated (4). Asch et al. report that the phosphorylation state of the extracellular threonine 92 may determine the relative binding affinity of CD36 for TSP-1 and collagen (5).

A well-defined human blood group polymorphism, $\mathrm{Nak}^{\mathrm{a}}$, is carried by platelet CD36 (6). The null phenotype ( $\mathrm{Nak}^{\mathrm{a}}$-negative) occurs with high frequency in African, Japanese, and other Asian populations, and this is perhaps related to the role of CD36 in malaria pathogenesis. Affected individuals may lack platelet (type II deficiency) or both platelet and monocyte CD36 (type I). The genetic basis of the Naka negative phenotype is under study by several groups.

CD36 protein sequence is highly conserved between the cloned human and rodent proteins. There are two CD36-family homologs in Drosophila, Croquemort, and epithelial membrane protein $(e m p)(7,8)$. Croquemort ("catcher of death") has 23\% homology to human CD36 at the amino acid level and is expressed on macrophages and hemocytes, where it is essential for phagocytosis of apoptotic corpses. Emp, which is expressed in precursor cells for adult epidermal structures, has 32\% homology to human CD36 and 34\% homology to human LIMP-II. There are three predicted CD36-family homologs in the genome of Caenorbabditis elegans. Study of the function of CD36 in these organisms provides insight into its primary human role and may also elucidate how this protein evolved to acquire its broad ligand specificity.

Structure-function relationships

The use of glutathione-S-transferase human CD36 fusion proteins and synthetic peptides has provided 
some basic structure-function information. Amino acids 93-120 define a minimal high-affinity binding site for TSP-1 that may be modulated by a downstream sequence (amino acids 139-155). Binding of oxidized LDL (oxLDL) has been mapped to a major domain at amino acids $120-155$ and a second region with less but significant affinity at amino acids 28-93. The site for binding of apoptotic cells and P. falciparum-infected erythrocytes is proposed to lie between amino acids 155 and 183, but this conclusion is based solely on $\mathrm{mAb}$ inhibition. Antibodies to this immunodominant epitope block all known CD36 function, including TSP-1 binding, making it difficult to interpret these results unequivocally.

Our laboratory determined that the TSP-1 binding site of CD36 is contained within a highly conserved sequence in $\mathrm{CD} 36$ family members that we termed the CLESH (CD36 LIMP-II Emp sequence homology) domain (9), which is also found in other proteins. Interestingly, the type I repeat of TSP-1, the domain containing the CD36 binding site, is also found in many other proteins. Characterizing expression profiles of these domains may identify potential receptorligand pairs. For example, we noted that the gp120 envelope protein of HIV contains a CLESH domain and showed that it binds TSP-1 (10). This knowledge may directly relate to the inhibitory effect on HIV infectivity of saliva, which contains a large concentration of TSP-1, and it may be exploited in the design of drugs to inhibit HIV transmission.

\section{Distribution and function of CD36}

CD36 colocalizes with caveolin-1 in specialized plasma membrane microdomains known as caveolae (11). These cholesterol- and sphingolipid-enriched structures may serve to concentrate signaling molecules and facilitate the integration of signaling cascades. For example, in platelets, in addition to CD36, src, and the src-related kinase lyn, also colocalize in these domains (12). Multiple lines of evidence indicate that caveolae serve an integral role in the trafficking of cholesterol in cells. Uittenbogaard et al. have shown that oxLDL depletes endothelial cell caveolae of cholesterol, resulting in displacement of endothelial nitric oxide synthase and an altered response to acetylcholine (13). These disruptive effects of oxLDL are mediated by CD36 and can be blocked by interaction of SR-BI with HDL, which prevents the cholesterol depletion of caveolae. Recently, Lee et al. reported that CD36 and other dually acylated proteins that are targeted to caveolae can inhibit phosphorylation of caveolin-1 (14), suggesting a novel lipid-based regulatory mechanism that may affect downstream signaling events. Thus, there are three potential roles for CD36 in these domains: as a receptor that targets specific ligands to these structures, as a signaling molecule, and as a regulator of caveolar function.

CD36 expression is broad and includes microvascular (but not large vessel) endothelium, adipocytes, skeletal muscle, dendritic cells, epithelia of the retina, breast, and intestine, smooth muscle cells, and hematopoietic cells, including erythroid precursors, platelets, monocytes/macrophages, and megakaryocytes. Evidence would suggest that scavenger function related to innate immunity is the most ancient role of CD36, but two other important activities have emerged: fatty acid transport and regulation of angiogenesis.

\section{Uptake of modified lipoproteins:}

\section{CD36 in atherogenesis}

An important pathologic function of scavenger receptors, related to macrophage foam cell formation and the pathogenesis of atherosclerosis, is recognition and internalization of oxidatively modified LDL. In 1993, Endemann et al. first identified CD36 as a potential oxLDL receptor (15). Unlike macrophage scavenger receptor A types I and II (SR-AI/II), CD36 binds LDL that has been exposed to "minimally" oxidizing conditions. Later work by Podrez et al. showed that, again in contrast to SR$\mathrm{AI} / \mathrm{II}, \mathrm{CD} 36$ can recognize LDL modified by the myeloperoxidase-hydrogen peroxide-nitrite system of phagocytic cells (MPO-oxLDL), which may have more physiological relevance than copper-oxidized or acetylated LDL (16). The same authors have also shown that MPO-oxLDL-dependent foam cell formation can be inhibited by as much as $80 \%$ with mAb's against CD36 (17). Data from $\mathrm{Nak}^{\mathrm{a}}$-negative individuals further support a role for CD36 in foam cell formation: Incubation of CD36-deficient monocytes/macrophages with oxLDL results in only $40-60 \%$ as much oxLDL binding, internalization, and cholesterol ester accumulation as is seen in CD36-expressing cells. The most compelling data supporting a critical role for CD36 in foam cell formation and atherosclerosis are from studies of a CD36-null mouse engineered by our laboratory. Macrophages isolated from these animals are profoundly defective in uptake of oxLDL and foam cell formation. We have found that breeding the CD36 deficiency onto a proatherogenic apoE-null background yields animals that are significantly protected from lesion development. Animals fed a Western diet showed a $>70 \%$ reduction in aortic lesion size and distribution (18).

Clearance of apoptotic cells: role in infection, immunity, and retina homeostasis

The first identification of CD36 scavenger receptor function was made by Savill et al., who were investigating recognition of apoptotic cells by mononuclear phagocytes (19). There are several receptor systems involved in this process, one of which involves CD36 in cooperation with $\alpha_{v} \beta_{3}$ integrin on the phagocyte surface. The ligand on the surface of the apoptotic cell varies, but the anionic phospholipid phosphatidylserine has been implicated in this process, as have modified lipids and TSP-1. Steinberg and others have postulated that the primary function of scavenger receptors is clearance of apoptotic cells arising during development, normal homeostasis, and infection (20). The characterization of Croquemort as a mediator of apoptotic cell clearance in Drosophila development supports this hypothesis. Witztum and colleagues (21) have shown that ligands on apoptotic cells cross-react with antibodies raised against epitopes on oxLDL, and this may explain some physical characteristics of scavenger receptor ligand recognition. Incubation of 
macrophages with apoptotic cells leads to increased expression of inhibitory mediators of inflammation and decreased LPS-mediated secretion of proinflammatory mediators. Some of these effects can be blocked with antibodies against TSP-1 or can be reproduced by substituting ligation of CD36 for coincubation with apoptotic cells. These findings suggest that signals mediated by CD36 ligation are responsible for these anti-inflammatory effects, and they reveal an interesting parallel between monocyte/macrophage and endothelial CD36 signaling: in both circumstances agonist-induced activation pathways (bFGF or LPS) are downmodulated.

Two interesting variations of apoptotic cell clearance, photoreceptor segment catabolism and antigen "crosspriming" (see below), are also mediated by CD36. The retinal pigment epithelium (RPE) envelops photoreceptor rod outer segments (ROSs), which are shed daily and must be phagocytosed and degraded to maintain normal vision. ROS membranes are composed of rhodopsin and phospholipids, including anionic phospholipids, which have been shown to be CD36 ligands. Our studies suggest that CD36 is at least one of the receptors involved in ROS recognition and internalization by RPE (22), another being the integrin $\alpha_{v} \beta_{5}$. Thus, as in the uptake of apoptotic cells, photoreceptor segment phagocytosis involves CD36, an $\alpha_{v}$ integrin, and the ligand phosphatidylserine.

Recognition and internalization by dendritic cells (DCs) of apoptotic cells that result from malignant transformation or viral infection can lead to "crosspriming" - presentation of tumor or viral antigens to $\mathrm{CD}^{+} \mathrm{T}$ cells in the context of MHC-I antigen. Albert et al. have shown that the recognition and uptake of apoptotic cells by immature dendritic cells and subsequent cross-priming are mediated by CD36 and $\alpha_{v} \beta_{5}(23)$. This process bears striking resemblance to the function of CD36 in macrophages but results in antigen representation instead of degradation. These studies support the hypothesis of Krieger and colleagues that scavenger receptors originated from the innate immune system as recognition molecules for foreign antigens (24).

Interaction with modified erythrocytes:

possible role in the pathogenesis

of malaria and sickle cell disease

The pathophysiological event that distinguishes $P$. falciparum malaria is adherence of parasitized erythrocytes to the microvasculature of key organs, especially in the brain, a process known as cytoadherence. CD36-dependent cytoadhesion, a property of almost all $P$. falciparum malaria isolates, involves recognition of membrane changes induced by parasitic infection (25). Interestingly, two studies have found little cerebral expression of CD36, and thus its role in P. falciparum morbidity has been questioned. It has been suggested that cytoadhesion can also sequester parasitized erythrocytes and allow their escape from immune surveillance. In Africa, there is a high frequency of mutation in the $C D 36$ gene; the most common mutation is predicted to cause premature truncation of the protein upstream of the reported binding site for parasitized erythrocytes. Aitman et al. tested the hypothesis that CD36 deficiency correlates with less severe malaria, as defined by cerebral involvement (26). They found, in fact, the opposite: Those patients with CD36 deficiency had more severe malaria, suggesting that expression of macrophage CD36 provides an important clearance mechanism for parasitized erythrocytes and prevents overwhelming infection. Similarly, McGilvray et al. recently showed that macrophage CD36 mediates nonopsonic phagocytosis of $P$. falciparum-infected erythrocytes (27). Phagocytosis is independent of $\alpha_{w} \beta_{3}$ and TSP- 1 and is probably mediated by CD36 recognition of a parasite-encoded ligand with homology to TSP-1. Although these studies support the hypothesis that scavenger receptors originally functioned as part of the innate immune system, the question of why CD36 deficiency has been maintained in Africa and Asia remains unanswered.

CD36 may also recognize alterations in erythrocyte membranes resulting from hemoglobin $\mathrm{S}$, another mutation prevalent in African populations. The slower transit time of sickle cells promotes adhesive interactions involving multiple receptor/ligand complexes, including CD36-TSP-1, contributing to the vascular pathobiology of the disease (28). TSP-1 may act as a bridging molecule between $\mathrm{CD} 36$ on the reticulocyte surface and CD36 on the endothelium to bring about adhesion. Additionally, sickle erythrocytes are characterized by membrane asymmetry, a hallmark of apoptotic cells, which may further promote recognition by CD36. Interestingly, heterozygosity for hemoglobin $S$ protects against $P$. falciparum malaria because the parasites cannot invade reticulocytes. The persistence of both these mutations at high frequency in Africa may result from complex evolutionary pressures related to malaria.

CD36 as a transporter of long-chain fatty acids Studying rat adipocytes, Abumrad identified CD36 as a fatty acid translocase (FAT) necessary for the transport of long-chain fatty acids (LCFAs) (29). Proteinfacilitated transport of LCFAs has been controversial, because data suggest that they can diffuse across cell membranes. However, molecules such as glucose can also freely diffuse, but regulated transport by protein mediators has been unquestionably established. Part of the contentiousness relates to the use of the word "transporter" or "translocator," which may imply a classic energy-dependent receptor mediated process. Although a mechanism has not been defined, and it is not known whether CD36 affects this function alone or with other proteins in a complex, the experimental data support a role for CD36 in regulated LCFA uptake in a variety of tissues. Recent studies on CD36 transgenic and null mice have confirmed a physiologic role for fatty acid transporters.

CD36 facilitates fatty acid translocation not just in adipocytes, but also in heart and skeletal muscle, where LCFAs are important substrates for energy production. Transgenic mice engineered to overexpress CD36 in skeletal muscle showed enhanced fatty acid oxidation, decreased circulating fatty acids and triacylglycerols, and decreased fat deposition (30). CD36- 


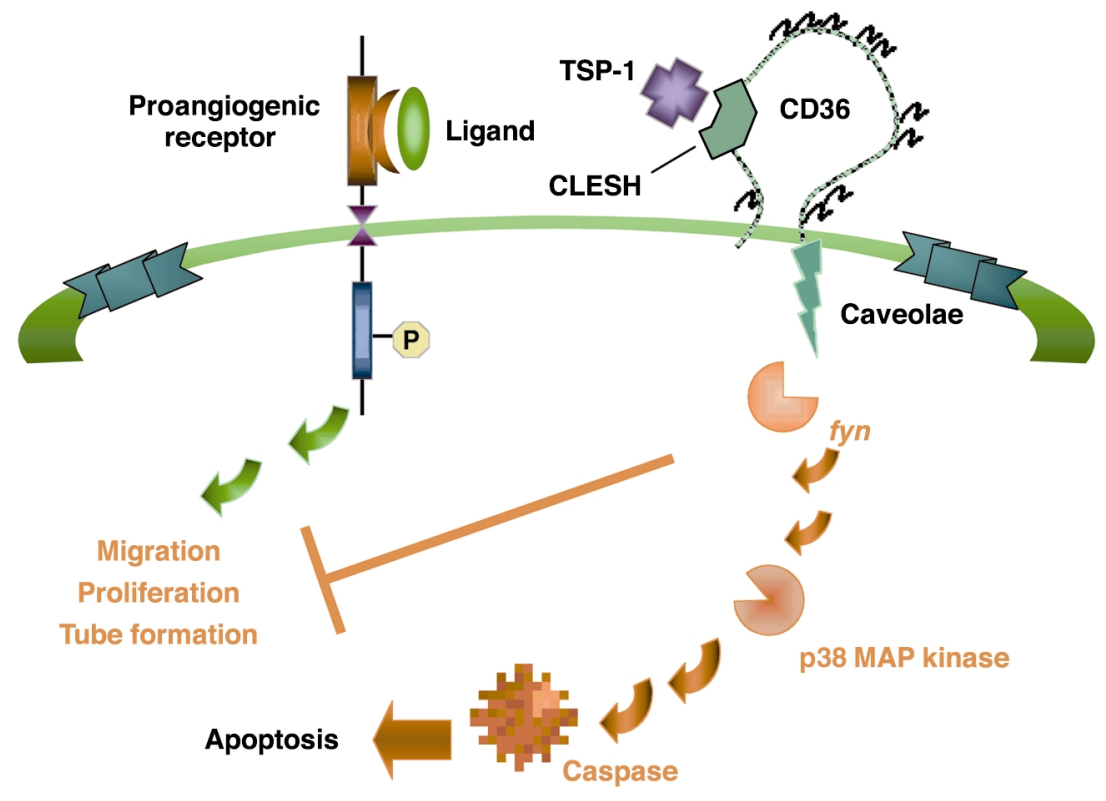

Figure 1

Antagonism of proangiogenic responses by CD36. In response to ligand engagement, a proangiogenic receptor (such as the VEGF or bFGF receptor) induces proliferation, migration, and tube formation of microvascular endothelial cells. In the presence of TSP-1, which interacts with a specific motif (CLESH) on its receptor, CD36, this response is inhibited. Inhibition is mediated through interaction with fyn, the activation of p38 MAP kinase, and, ultimately, caspase DNA cleavage, resulting in endothelial cell apoptosis.

null mice had decreased adipocyte and oxidative muscle transport of LCFAs, increased levels of cholesterol, triacylglycerol and fatty acids, and fasting hypoglycemia (31). These changes reflect an impact on energy utilization from LCFAs and suggest an important role for CD36 in lipoprotein and lipid homeostasis. Further in vivo support has come from studies of the spontaneous hypertensive rat (SHR), which has defective adipocyte transport of LCFAs and a type 2 diabetes syndrome and was shown to have a mutant CD36 gene (32). Finally, studies in humans have also provided support for a physiological role for CD36 in LCFA translocation. Lack of uptake of a LCFA analog in heart correlates with the Nak ${ }^{a}$ negative phenotype, and some studies show a correlation with diabetes or cardiomyopathy (33). These latter reports remain controversial because of the small cohort sizes.

\section{CD36 and the antiangiogenic activity of TSP-1}

The work of Bouck and colleagues identified TSP-1 and derived fragments as potent endogenous inhibitors of tumor angiogenesis, effective against virtually all angiogenic inducers including bFGF and VEGF (34). Further study demonstrated that the domain of TSP-1 responsible for this angiostatic effect lies within the type I repeat, suggesting that $\mathrm{CD} 36$ may be involved. Our laboratory subsequently confirmed that CD36 mediates the antiangiogenic signal of TSP-1 (35). In recent studies, Simantov et al. showed that this interaction can be modulated by another CLESH-containing protein, histidine-rich glycoprotein (36). With Bouck and colleagues, we have begun to delineate the pathway by which TSP-1/CD36 exerts an antiangiogenic response (37). A model of this pathway is shown in Figure 1. Studies in several cell types have shown that CD36 physically associates with nonreceptor protein tyrosine kinases fyn, lyn, and yes. Exposure of microvascular endothelial cells (ECs) to TSP-1 leads to recruitment of fyn to a CD36 membrane complex with activation of the kinase and subsequent downstream activation of p38 mitogenactivated protein kinase. Studies with cells from null animals and pharmacological and immunologic inhibitors have shown that the antiangiogenic signal mediated by CD36 and TSP-1 is dependent on both of these kinases. Furthermore, the antiangiogenic activity of TSP1 is linked to its ability to induce EC apoptosis via caspase-3-like effectors. CD36 signaling can lead to programmed cell death in other cell types: Rusinol et al. showed that incubation of CD36-transfected Chinese hamster ovary cells with oxLDL resulted in a dose- and timedependent induction of apoptosis, which did not occur in nontransfected cells (38). As shown in the model, we suggest that the CD36/TSP-1 antiangiogenic signal provides a "switch" that diverts a growth factor-mediated proangiogenic response to an antiangiogenic, proapoptotic re-sponse. Unraveling this antagonistic pathway and comparing it with those of other angiogenic inhibitors may reveal a common element which would provide a powerful therapeutic target that can be exploited in the treatment of tumors, diabetic retinopathy, and other diseases in which angiogenesis is a component.

\section{CD36 ligands}

Scavenger receptors have high affinities for modified lipoproteins (see Platt and Gordon [ref. 39] and Krieger [ref. 40], both in this Perspective series). In the class B family, nothing is known of the ligands for LIMP-II, but those for CD36 and SR-BI have been well defined. SR-BI and CD36 have both been reported to bind native and modified lipoproteins, but it is interesting to note how their functions have diverged in the course of evolution. The interaction between SR-BI and HDL has become exquisitely refined to allow for selective lipid uptake and lipid exchange between cells and HDL (41). In contrast, CD36 has emerged as a specific receptor for transport of LCFAs. Part of this refinement of function may pertain to their respective tissue expression patterns. While SR-BI is predominantly expressed in steroidogenic tissues and liver, CD36 is expressed in tissues with high LCFA storage or oxidative capacity. Additionally, the expression of CD36 on macrophages, $\mathrm{RPE}$, and DCs has possibly resulted in retention of 
some primitive scavenger functions, such as uptake of apoptotic cells. An extension of this role is recognition of sickle and parasitized erythrocytes, which have features in common with apoptotic cells. The ability of CD36 to cooperate in cross-priming in DCs shows evolution of the innate function of simple recognition of pathogen-induced apoptotic cells or host cells rendered "foreign" by mutation. Whether recognition of oxLDL is a further evolution of the capacity to recognize ligands common to apoptotic cells remains an open question, but the pathology that has emerged from this interaction is largely attributable to the Western diet.

\section{Regulation of CD36 expression: identification}

of a proatherogenic feed-forward loop

Our laboratory and others have demonstrated that CD36 expression is highly regulated in monocytes and that it can be upregulated at the transcriptional level by adhesion, M-CSF, GM-CSF, native and modified LDLs, cellular cholesterol, and IL-4. CD36 is downregulated by corticosteroids, TGF- $\beta 1$, HDL, and LPS (42-44), consistent with the idea that CD36 expression correlates with monocyte maturation and is downregulated during inflammation. A critical regulator of CD36 expression is the nuclear hormone receptor PPAR $\gamma(45,46)$. The human $C D 36$ gene contains a PPAR $\gamma$-responsive element shown to be functional in macrophages. Ligands for $\operatorname{PPAR} \gamma$ (which include lipids derived from oxLDL, 9- and 13-hydroxyoctadecadienoic acid [HODE], prostaglandin J2 [PGJ2], and the thiazolidinedione class of antidiabet- ic drugs) increase CD36 expression. The induction of CD36 by IL- 4 in macrophages was recently shown by Huang et al. to be mediated by activation of intracellular lipoxygenases and generation of the PPAR $\gamma$ ligand PGJ2 (47). Downregulation of CD36 by TGF- $\beta$ was observed to be related to phosphorylation and inactivation of PPAR $\gamma$. The relationship between CD36 expression and PPAR $\gamma$, as well as the ability of CD36 to participate in macrophage internalization of potential lipid ligands for PPAR $\gamma$, led Evans and colleagues to postulate a proatherogenic feed-forward loop in the vessel wall (46). Figure 2 shows a model for this pathway, in which macrophages and LDL particles become entrapped in the vessel wall as a result of a proatherogenic injury (48). Inflammatory stimuli then lead to production of reactive oxygen and nitrogen metabolites, generating epitopes on LDL that are recognized by CD36. This leads to internalization of modified LDL, generation of intracellular PPAR $\gamma$ ligands, translocation of active PPAR $\gamma$ transcriptional activator complexes to the nucleus, and upregulation of PPAR $\gamma$ target genes, including PPAR $\gamma$ itself and CD36. The increased expression of CD36 on the cell surface leads to further internalization of modified LDL and eventual foam cell formation.

In heart and skeletal muscle, CD36 is regulated by plasma triacylglycerol and fatty acid levels and energy requirements. Cardiac CD36 expression is also responsive to an agonist of PPAR $\alpha$ but not PPAR $\gamma$, which reflects the cellular distribution of these factors, and establishes a pattern of regulation of CD36 by this fam-

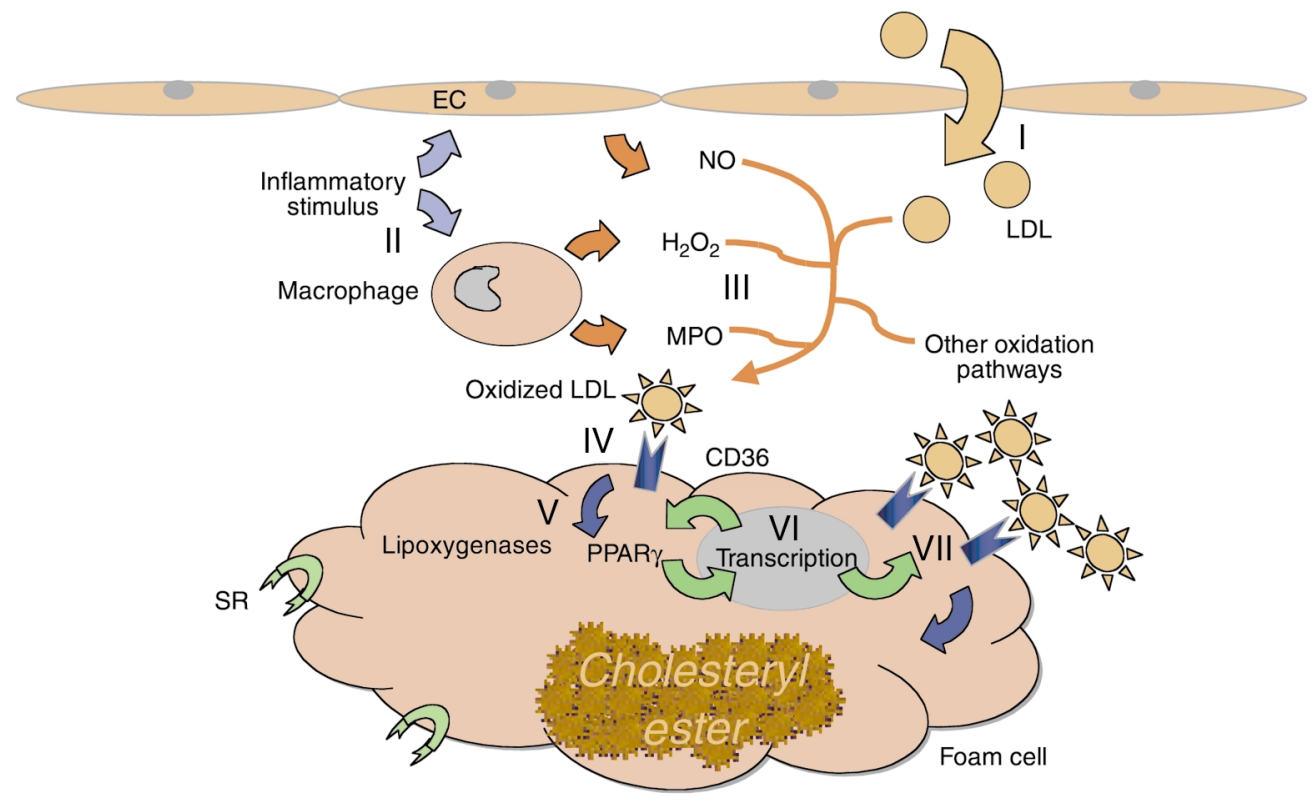

\section{Figure 2}

CD36-mediated macrophage foam cell formation. In response to EC injury, macrophages and LDL transmigrate into the subendothelial space (I), where they may become entrapped. Inflammatory stimuli (II) lead to the secretion of oxidative products from ECs and macrophages, including nitric oxide (NO), hydrogen peroxide, and myeloperoxidase (MPO) (III), which act upon LDL particles and convert them into CD36-specific ligands, or ligands for other scavenger receptors (SR). Internalization of oxidized LDL via CD36 (IV) generates lipid byproducts (e.g., 9-HODE, 13-HODE, and PGJ2), mediated by lipoxygenase or other pathways, which in turn provide ligands for the transcription factor PPAR $\gamma(V)$. The binding of these lipids structurally enables PPAR $\gamma$ to dimerize with binding partners such as RXR and charges the complex for nuclear translocation and the activation of transcription of target genes $(\mathrm{VI})$. Because these target genes include both PPAR $\gamma$ and $C D 36$, a positive feedback loop arises. The increased expression of CD36 promotes further oxidized LDL uptake, perpetuating the cycle and resulting in accumulation of cholesterol ester by macrophages and eventually in foam cell formation (VII). Adapted from ref. 48. 
ily. During adipocyte development, CD36 expression increases as the cells become competent to take up and store LCFAs and may be essential to maturation (49). Adipogenesis is accompanied by expression of several members of the PPAR family, including PPAR $\gamma 2$ and $\operatorname{PPAR} \alpha(\delta)$, which may regulate CD36 expression. Expression of CD36 and other fatty acid transporters is also regulated in response to environmental cues, such as plasma insulin and glucose, LPS, and cytokines.

\section{Possible link to insulin resistance}

The expression and regulation of CD36 in adipose tissue, skeletal muscle, and heart and its role as a translocator of LCFAs position it as a potential mediator of energy metabolism and may suggest an indirect role in glucose uptake and utilization. This hypothesis is supported by work with a diabetic strain of SHR by Aitman et al. (32), who showed that these animals express a mutant $C D 36$ gene. Replacement of the mutant allele with the wild-type version in a congenic strain reverses the fatty acid transport defect and corrects the diabetic phenotype. Further support for a role for $\mathrm{CD} 36$ in glucose metabolism comes from studies showing a higher prevalence of type 2 diabetes in $\mathrm{Nak}^{\mathrm{a}}$-negative patients. Interestingly, CD36-null mice are not diabetic, perhaps reflecting species differences in glucose and fatty acid metabolism or a difference in genetic modifiers. However, CD36-null mice do have altered lipid and glucose metabolism and provide a model to dissect the interrelationship between CD36, fatty acid and glucose uptake and utilization. Interestingly, Li et al. reported that treatment of LDL receptor-deficient mice with thiazolidinediones improves insulin sensitivity in male (but not female) mice. In spite of the impact these drugs have on macrophage CD36 expression, they appear to decrease atherosclerosis in male mice (50). It may be that insulin resistance has the greatest proatherogenic effect in this model and that correction of this metabolic feature counters any negative effect due to potential upregulation of macrophage CD36. This study therefore underscores the complexity of the regulation of CD36 in different tissues and in response to different environmental cues.

\section{Conclusion}

Work in the last 25 years has established CD36 as a multiligand receptor involved in cellular adhesion; fatty acid and lipid transport, utilization, and storage; antigen presentation; and clearance of apoptotic cells and shed photoreceptors. The challenge for the future will be to understand the mechanisms by which it effects these diverse functions and to design therapeutic strategies that can alter the course of the diseases with which it is associated, including atherosclerosis, diabetes, cardiomyopathy, obesity, blindness, sickle cell anemia, and malaria.

\section{Acknowledgments}

Reference space limitations preclude recognition of many colleagues who have contributed to our knowledge of CD36; we wish to acknowledge them here. We wish to thank members of the Silverstein laboratory, especially David Lennon and Ronit Simantov, and David Stern for critical reading of this manuscript and insightful suggestions. The studies summarized here were supported in part by grants from the NIH (EY-10967, HL-42540, HL-58559, POHL-56987), the Silbermann Fellowship, and the Dorothy Rodbell Cohen Foundation.

1. Adams, J.C., Tucker, R.P., and Lawler, J. 1995. The thrombospondin gene family. R.G. Landes. Austin, Texas, USA.

2. Silverstein, R.L., and Febbraio, M. 2000. CD36 and atherosclerosis. Curr. Opin. Lipidol. 11:483-491.

3. Oquendo, P., Hundt, E., Lawler, J., and Seed, B. 1989. CD36 directly mediates cytoadherence of Plasmodium falciparum parasitized erythrocytes. Cell. 58:95-101.

4. Gruarin, P., et al. 2000. CD36 is a ditopic glycoprotein with the N-terminal domain implicated in intracellular transport. Biochem. Biophys. Res. Commun. 275:446-454.

5. Asch, A.S., et al. 1993. Analysis of CD36 binding domains: ligand specificity controlled by dephosphorylation of an ectodomain. Science. 262:1436-1440.

6. Yamamoto, N., et al. 1990. A platelet membrane glycoprotein (GP) deficiency in healthy blood donors: Naka- platelets lack detectable GPIV (CD36). Blood. 76:1698-1703.

7. Franc, N.C., Dimarcq, J.L., Lagueux, M., Hoffmann, J., and Ezekowitz, R.A. 1996. Croquemort, a novel Drosophila hemocyte/macrophage receptor that recognizes apoptotic cells. Immunity. 4:431-443.

8. Hart, K., and Wilcox, M. 1993. A Drosophila gene encoding an epithelial membrane protein with homology to CD36/LIMP II. J. Mol. Biol. 234:249-253.

9. Crombie, R., and Silverstein, R.L. 1998. Lysosomal integral membrane protein II binds thrombospondin-1. Structure-function homology with the cell adhesion molecule CD36 defines a conserved recognition motif. J. Biol. Chem. 273:4855-4863.

10. Crombie, R., et al. 1998. Identification of a CD36-related thrombospondin 1-binding domain in HIV-1 envelope glycoprotein gp120: relationship to HIV-1-specific inhibitory factors in human saliva. J. Exp. Med. 187:25-35.

11. Lisanti, M.P., et al. 1994. Characterization of caveolin-rich membrane domains isolated from an endothelial-rich source: implications for human disease. J. Cell Biol. 126:111-126.

12. Dorahy, D.J., Lincz, L.F., Meldrum, C.J., and Burns, G.F. 1996. Biochemical isolation of a membrane microdomain from resting platelets highly enriched in the plasma membrane glycoprotein CD36. Biochem. J. 319:67-72.

13. Uittenbogaard, A., Shaul, P.W., Yuhanna, I.S., Blair, A., and Smart, E.J. 2000. High density lipoprotein prevents oxidized low density lipoprotein-induced inhibition of endothelial nitric-oxide synthase localization and activation in caveolae. J. Biol. Chem. 275:11278-11283.

14. Lee, H., et al. 2001. Palmitoylation of caveolin-1 at a single site (Cys-156) controls its coupling to the c-Src tyrosine kinase. J. Biol. Chem. In press. Published July 12, 2001 as 10.1074 /jbc.M104530200.

15. Endemann, G., et al. 1993. CD36 is a receptor for oxidized low density lipoprotein. J. Biol. Chem. 268:11811-11816.

16. Podrez, E.A., Schmitt, D., Hoff, H.F., and Hazen, S.L. 1999. Myeloperoxidase-generated reactive nitrogen species convert LDL into an atherogenic form in vitro. J. Clin. Invest. 103:1547-1560.

17. Podrez, E.A., et al. 2000. Macrophage scavenger receptor CD36 is the major receptor for LDL modified by monocyte-generated reactive nitrogen species. 105:1095-1108.

18. Febbraio, M., et al. 2000. Targeted disruption of the class B scavenger receptor CD36 protects against atherosclerotic lesion development in mice. J. Clin. Invest. 105:1049-1056.

19. Savill, J., Hogg, N., and Haslett, C. 1991. Macrophage vitronectin receptor, CD36, and thrombospondin cooperate in recognition of neutrophils undergoing programmed cell death. Chest. 99:6S-7S.

20. Bird, D.A., et al. 1999. Receptors for oxidized low-density lipoprotein on elicited mouse peritoneal macrophages can recognize both the modified lipid moieties and the modified protein moieties: implications with respect to macrophage recognition of apoptotic cells. Proc. Natl. Acad. Sci. USA. 96:6347-6352.

21. Chang, M.K., et al. 1999. Monoclonal antibodies against oxidized lowdensity lipoprotein bind to apoptotic cells and inhibit their phagocytosis by elicited macrophages: evidence that oxidation-specific epitopes mediate macrophage recognition. Proc. Natl. Acad. Sci. USA. 96:6353-6358.

22. Ryeom, S.W., Sparrow, J.R., and Silverstein, R.L. 1996. CD36 participates in the phagocytosis of rod outer segments by retinal pigment epithelium. J. Cell Sci. 109:387-395.

23. Albert, M.L., et al. 1998. Immature dendritic cells phagocytose apoptotic cells via alphavbeta5 and CD36, and cross-present antigens to cytotoxic T lymphocytes. J. Exp. Med. 188:1359-1368. 
24. Krieger, M. 1997. The other side of scavenger receptors: pattern recognition for host defense. Curr. Opin. Lipidol. 8:275-280.

25. Ockenhouse, C.F., et al. 1991. Molecular basis of sequestration in severe and uncomplicated Plasmodium falciparum malaria: differential adhesion of infected erythrocytes to CD36 and ICAM-1.J. Infect. Dis. 164:163-169.

26. Aitman, T.J., et al. 2000. Malaria susceptibility and CD36 mutation. Nature. 405:1015-1016

27. McGilvray, I.D., Serghides, L., Kapus, A., Rotstein, O.D., and Kain, K.C 2000. Nonopsonic monocyte/macrophage phagocytosis of plasmodium falciparum-parasitized erythrocytes: a role for CD36 in malarial clearance. Blood. 96:3231-3240.

28. Sugihara, K., Sugihara, T., Mohandas, N., and Hebbel, R.P. 1992. Thrombospondin mediates adherence of $\mathrm{CD} 36+$ sickle reticulocytes to endothelial cells. Blood. 80:2634-2642.

29. Abumrad, N.A., el-Maghrabi, M.R., Amri, E.Z., Lopez, E., and Grimaldi, P.A. 1993. Cloning of a rat adipocyte membrane protein implicated in binding or transport of long-chain fatty acids that is induced during preadipocyte differentiation. Homology with human CD36. J. Biol. Chem. 268:17665-17668.

30. Ibrahimi, A., et al. 1999. Muscle-specific overexpression of FAT/CD36 enhances fatty acid oxidation by contracting muscle, reduces plasma triglycerides and fatty acids, and increases plasma glucose and insulin. J. Biol. Chem. 274:26761-26766.

31. Febbraio, M., et al. 1999. A null mutation in murine CD36 reveals an important role in fatty acid and lipoprotein metabolism. J. Biol. Chem. 274:19055-19062.

32. Aitman, T.J., et al. 1999. Identification of Cd36 (Fat) as an insulin-resistance gene causing defective fatty acid and glucose metabolism in hypertensive rats. Nat. Genet. 21:76-83.

33. Hwang, E.H., et al. 1998. Absent myocardial iodine-123-BMIPP uptake and platelet/monocyte CD36 deficiency. J. Nucl. Med. 39:1681-1684.

34. Good, D.J., et al. 1990. A tumor suppressor-dependent inhibitor of angiogenesis is immunologically and functionally indistinguishable from a fragment of thrombospondin. Proc. Natl. Acad. Sci. USA. 87:6624-6628.

35. Dawson, D.W., et al. 1997. CD36 mediates the in vitro inhibitory effects of thrombospondin-1 on endothelial cells. J. Cell. Biol. 138:707-717.

36. Simantov, R., et al. 2001. Histidine rich glycoprotein inhibits the antiangiogenic effect of thrombospondin-1. J. Clin. Invest. 107:45-52.

37. Jimenez, B., et al. 2000. Signals leading to apoptosis-dependent inhibition of neovascularization by thrombospondin-1. Nat. Med. 6:41-48.
38. Rusinol, A.E., et al. 2000. Isolation of a somatic cell mutant resistant to the induction of apoptosis by oxidized low density lipoprotein. J. Biol. Chem. 275:7296-7303.

39. Platt, N., and Gordon, S. 2001. Is the class A macrophage scavenger receptor (SR-A) multifunctional? - The mouse's tale. J. Clin. Invest. 108:649-654

40. Krieger, M. 2001. Scavenger receptor class B type I is a multiligand HDL receptor that influences diverse physiologic systems. J. Clin. Invest. 108:793-797.

41. Acton, S., et al. 1996. Identification of scavenger receptor SR-BI as a high density lipoprotein receptor. Science. 271:518-520.

42. Huh, H.Y., Pearce, S.F., Yesner, L.M., Schindler, J.L., and Silverstein, R.L. 1996. Regulated expression of CD36 during monocyte-to-macrophage differentiation: potential role of CD36 in foam cell formation. Blood. 87:2020-2028.

43. Han, J., et al. 2000. Transforming growth factor-beta1 (TGF-beta1) and TGF-beta2 decrease expression of CD36, the type B scavenger receptor, through mitogen-activated protein kinase phosphorylation of peroxisome proliferator-activated receptor-gamma. J. Biol. Chem. 275:1241-1246.

44. Feng, J., et al. 2000. Induction of CD36 expression by oxidized LDL and IL-4 by a common signaling pathway dependent on protein kinase $\mathrm{C}$ and PPAR-gamma. J. Lipid Res. 41:688-696.

45. Nagy, L., Tontonoz, P., Alvarez, J.G., Chen, H., and Evans, R.M. 1998. Oxidized LDL regulates macrophage gene expression through ligand activation of PPAR gamma. Cell. 93:229-240.

46. Tontonoz, P., Nagy, L., Alvarez, J.G., Thomazy, V.A., and Evans, R.M. 1998. PPARgamma promotes monocyte/macrophage differentiation and uptake of oxidized LDL. Cell. 93:241-252.

47. Huang, J.T., et al. 1999. Interleukin-4-dependent production of PPARgamma ligands in macrophages by $12 / 15$-lipoxygenase. Nature. 400:378-382.

48. Steinberg, D. 1997. Lewis A. Conner Memorial Lecture. Oxidative modification of LDL and atherogenesis. Circulation. 95:1062-1071.

49. Sfeir, Z., Ibrahimi, A., Amri, E., Grimaldi, P., and Abumrad, N. 1997. Regulation of FAT/CD36 gene expression: further evidence in support of a role of the protein in fatty acid binding/transport. Prostaglandins Leukot. Essent. Fatty Acids. 57:17-21.

50. Li, A.C., et al. 2000. Peroxisome proliferator-activated receptor gamma ligands inhibit development of atherosclerosis in LDL receptor-deficient mice. J. Clin. Invest. 106:523-531. 